\title{
MMHealth 2017: Workshop on Multimedia for Personal Health and Health Care
}

\author{
Susanne Boll \\ University of Oldenburg, Germany \\ Susanne.Boll@uol.de \\ Laleh Jalali \\ Hitachi America Ltd., USA \\ lalehjal@gmail.com
}

\author{
Touradj Ebrahimi \\ EPFL, Switzerland \\ touradj.ebrahimi@epfl.ch \\ Ramesh Jain \\ UC Irvine, USA \\ jain@ics.uci.edu \\ Noel E. O’Connor \\ Dublin City University, Ireland \\ noel.oconnor@dcu.ie
}

\author{
Cathal Gurrin \\ Dublin City University, Ireland \\ cgurrin@computing.dcu.ie \\ Jochen Meyer \\ OFFIS-Institute for IT, Germany \\ meyer@offis.de
}

\begin{abstract}
Ever since the emergence of digitization, we've used the term multimedia to represent a combination of different kinds of media types, such as images, audio, and videos. As new sensing technologies emerge and are now becoming omnipresent in daily lives, the definition, role and significance of multimedia is changing. Multimedia now represents the means for communicating, cooperating, and also for monitoring numerous aspects of daily life, at various levels of granularity and application, ranging from personal to societal. With this shift, we have since moved from comprehending single media and its state toward comprehending media in terms of its use context.

Multimedia is thus no longer confined to documentation and preservation, entertainment or personal media collections; rather, it has become an integral part of the tools and systems that are providing solutions to today's societal challengesincluding challenges related to health, aging, education, societal participation, sustainable energy, and intelligent transportation. Multimedia has thus evolved into a core enabler for future interactive and cooperative applications at the heart of society. In this workshop we explore the relevance and contribution of multimedia to health care and personal media.
\end{abstract}

\section{CCS Concepts}

Human-centered computing, Consumer health, Health care information systems, Multimedia information systems, Digital signal processing

\section{Keywords}

Multimedia, health care, personal health

Permission to make digital or hard copies of part or all of this work for personal or classroom use is granted without fee provided that copies are not made or distributed for profit or commercial advantage and that copies bear this notice and the full citation on the first page. Copyrights for third-party components of this work must be honored. For all other uses, contact the Owner/Author.

MM '17, October 23-27, 2017, Mountain View, CA, USA

(C) 2017 Copyright is held by the owner/author(s).

ACM ISBN 978-1-4503-4906-2/17/10.

https://doi.org/10.1145/3123266.3132051

\section{BACKGROUND AND MOTIVATION}

Personal health and health care involves some of the state of the art technologies in multimedia. Multimedia technologies related to health and personal health can range from medical imaging to daily life monitoring. Medical imaging itself can range from molecular imaging that visualize biological processes, to $\mathrm{x}$-ray, ultrasound, MRI scans, CAT scans etc that visualize the internal body. In recent years, the rapidly increasing use of wearable and environmental sensors is providing a rich source of data describing numerous aspects of a person's daily life, behavior and lifestyle. Additionally, a great variety of wearable and ambient sensors have been developed and are constantly being improved and incorporated in peoples' daily lives, measuring physiological parameters in a non-intrusive and accurate manner, and producing new types of multimedia.

This data comes in a multitude of formats, targeted at virtually any type of device, thanks to efforts in web and mobile technology. As a result, electronic healthcare records (EHRs) are also adapting, to allow for the inclusion of medical imagery and other multimedia files, enabling the protection of privacy in their storage and transmission, while also allowing their rapid and reliable communication when needed. With the machine learning that were making large advancements in recent years, we will be able to provide holistic analysis and prediction of personal health and medical health at a scale that we have never seen before.

\section{WORKSHOP GOALS AND PROGRAM}

Research in multimedia and health is driven of the current technological advancements in sensors and personalised healthcare. In recent years we can observe many applications of health care and personal health that are addressed by core multimedia research questions, such as monitoring daily life activities, developing lifestyle and behavioral profiles, physiological and cognitive multimedia-based monitoring for health status assessment, among others. Applications can be as specific as the recognition of food to assess its nutritional content, multimodal visualization and correlation of lifestyle parameters to assess conditions such as dementia, or the development of personalized home assistants, used to help the elderly in their daily life, as society ages and the care ratio 
dwindles. There is an increasing number of research work that shows how core multimedia research is became an important enabler for applications with an application and relevance for the societal questions of health. The special characteristic of the workshop is the joining of a challenging and highly application domain and multimedia research. The workshop is of a very interdisciplinary nature which is reflected by its faceted topics of interest which are also covered by the different expertise of the organizers.

The special characteristic of the workshop is the joining of a challenging and highly application-oriented domain with more fundamental multimedia research and advances in the related technologies. The workshop is of a very interdisciplinary nature which is reflected by its faceted topics of contributions.

The workshop program reflects the interdisciplinary challenges in different fields of personal health and health care. The full paper program shows the broad and exciting scope of the field.

- Combining the benefits of CCA and SVMs for SSVEP-based BCIs in real-world conditions; Elisavet Chatzilari, Georgios Liaros, Kostas Georgiadis, Spiros Nikolopoulos and Yiannis Kompatsiaris (ITI-CERTH)

- Artificial Intelligence in XPRIZE DeepQ Tricorder; Edward Chang (HTC AI Research \& Healthcare); Meng-Hsi Wu (HTC AI Research \& Healthcare); Kai-Fu Tang, Hao-Cheng Kao, Chun-Nan Chou (HTC AI Research \& Healthcare)

- Gamification of Heel Raise Plantarflexion Physiotherapy; Jeannie Lee (Singapore Institute of Technology); Darren Goh (University of Glasgow); Alfred Tan (Singapore Institute of Technology)

- Research Challenges of Emerging Technologies Supporting Life-Long Health and Wellbeing; Jochen Meyer (OFFIS Institute for IT); Parisa Eslambolchilar (School of Computer Science and Informatics, Cardiff University)

- Empirical Mode Decomposition of Throat Microphone Recordings for Intake Classification; Mehmet Ali Tugtekin Turan and Engin Erzin ( Koc University)

- Wearable Emotion Recognition System based on GSR and PPG Signals; Goran Udovičić, Jurica Đerek, Mladen Russo and Marjan Sikora (University of Split, Faculty of Electrical Engineering, Mechanical Engineering and Naval Architecture)

- Denoising of Joint Tracking Data by Kinect Sensors using clustered Gaussian Process Regression; An-Ti Chiang and Yao Wang ( NYU)

- Live Personalized Nutrition Recommendation Engine; Nitish Nag, Vaibhav Pandey, Ramesh Jain (University of California, Irvine)

Additional short papers are complementing the workshop.

- Managing Family Healthcare with Multimedia Chat Apps: A Survey on What is Missing; Britta Meixner (CWI); Matthew Lee and Scott Carter (FXPAL)

- Toward Personalized Treatment of Chronic Diseases - the CKD Case Study; Chih-Yang Chen, Chun-Nan Chou (HTC AI Research \& Healthcare); I-Wen Wu (Department of Nephrology Keelung Chang Gung Memorial Hospital)

- DeepQ Arrhythmia Database: A Large-Scale Dataset for Arrhythmia Detector Evaluation; Meng-Hsi Wu and Edward Chang (HTC AI Research \& Healthcare)
- Exploring Challenges in Automated Just-In-Time Adaptive Food Choice Interventions; Nađa Terzimehić, Christina Schneegass and Heinrich Hussmann (Media Informatics Group, LMU Munich, Germany)

- Elderly Living Alone: Detecting Home Visits with Ambient and Wearable Sensing; Rui Hu, Hieu Pham, Philipp Buluschek, and Daniel Gatica-Perez (Idiap Research Institute)

- Few-shot Learning Using a Small-Sized Dataset of HighResolution FUNDUS Images for Glaucoma Diagnosis; Mijung Kim, Jasper Zuallaert and Wesley De Neve ( Ghent University)

- MedFit: a Mobile Application for Recovering CVD Patients; Jogile Kuklyte, Leonardo Gualano, Ghanashyama Prabhu, Kaushik Venkataraman, Deirdre Walsh, Catherine Woods, Kieran Moran and Noel E. O'Connor (The Insight Centre for Data Analytics, Dublin City University)

\section{ORGANIZERS}

The workshop is being held the second time. It bases not only on last year's experience MMHealth 2016 in Amsterdam but also on the strong research background and experience by the organizers in both multimedia and health. It is also based on previous events which have been organized by the organizers that strongly relate to multimedia and health. the authors were and are highly involved into several multimedia related international events in the field of the workshop: Measuring Behaviour 2016, PervasiveHealth 2014, SenseCam Symposium 2010, Beyond QuantifiedSelf at CHI 2014, among others.

The organizers are working in the different fields of multimedia in health such as tracking of nutrition[1], digital interventions for personal health [2], activity recognition from Smart phones [4] and in lifelogging [3] using statistical methods, from multimodal heterogeneous data [4] and interpretation for personal health [6].

\section{REFERENCES}

[1] A. Singla, L. Yuan and T. Ebrahimi. Food/Non-food Image Classification and Food Categorization using Pre-Trained GoogLeNet Model. 2nd Intl. Workshop on Multimedia Assisted Dietary Management, Amsterdam, The Netherlands, 2016.

[2] Jutta Fortmann, Vanessa Cobus, Wilko Heuten, Susanne Boll: WaterJewel: design and evaluation of a bracelet to promote a better drinking behaviour. MUM 2014: 58-67.

[3] Terziyski, Stefan and Albatal, Rami and Gurrin, Cathal (2015) Fast human activity recognition in lifelogging. MultiMedia Modeling, 8936 . pp. 369-379. ISSN 0302-9743

[4] Laleh Jalali, Hyungik Oh, Ramin Moazeni, and Ramesh Jain, "Human Behavior Analysis from Smartphone Data Streams", Human Behavior Understanding in ACM Multimedia, 2016.

[5] Hyungik Oh, Laleh Jalali, Ramesh Jain: An intelligent notification system using context from real-time personal activity monitoring. Multimedia and Expo (ICME'15), pp. 16, 2015

[6] Jochen Meyer, Merlin Wasmann, Wilko Heuten, Abdallah El Ali, and Susanne C.J. Boll. 2017. Identification and Classification of Usage Patterns in Long-Term Activity Tracking. In Proceedings of the 2017 CHI Conference on Human Factors in Computing Systems (CHI '17). ACM, New York, NY, USA, 66 